\title{
Visible signs of insulin resistance: opportunities lost
}

\author{
G. Nagamani • P. G. Sundararaman • G. R. Sridhar
}

Published online: 23 December 2014

(C) Research Society for Study of Diabetes in India 2014

The hope of a young physician's call for "Small fevers are gratefully accepted" can be matched by the despair of the senior physician's "If only diabetes were a little more symptomatic." The senior physicians' hopes can be fulfilled to a certain extent-if only.

While hypertension, hyperglycemia, and glaucoma may be asymptomatic, hirsutism and acne are not. Polycystic ovary syndrome (PCOS) is the most common endocrine disorder in women of reproductive age [1]. Of its manifestations, hyperandrogenism and infertility are the most prominent which bring young girls and women to medical attention. Being seen primarily by gynecologists and cosmetologists, the metabolic profile associated with PCOS lay silent. Symptomatic treatment for acne, hirsutism, or menstrual irregularity appeared to solve the problem.

It is now increasingly recognized that PCOS is a metabolic disorder with cosmesis and infertility being part of a spectrum that includes accelerated atherosclerosis, insulin resistance, coronary artery disease, obesity, and type 2 diabetes mellitus [1, 2]. The Androgen Excess Society criteria for diagnosis of PCOS included (a) hyperandrogenism, hirsutism and/or hyperandrogenemia; (b) ovarian dysfunction,

\section{G. Nagamani}

Department of Obstetrics and Gynecology, Andhra Medical College,

Visakhapatnam, India

P. G. Sundararaman

Apollo Hospitals, Off Greams Road, Chennai, India

G. R. Sridhar $(\bowtie)$

Endocrine and Diabetes Centre, Visakhapatnam, India

e-mail: sridharvizag@gmail.com oligo-anovulation and/or polycystic ovaries; and (c) exclusion of other androgen-excess or androgen-related disorders [3].

The recent position statement on PCOS from the European Society of Endocrinology [4] recognized its broad spectrum and emphasized the contributing roles of obesity; body composition; the occurrence of sleep disorders (that are related to obesity, insulin resistance, and diabetes mellitus) [5-7], with a separate section to cardiometabolic risk factors; and the need for targeting these along with infertility and hirsutism.

The primary reason why PCOS is brought to medical attention is because of hyperandrogenic features such as increased facial hair, acne, and loss of scalp hair [2]. The second presenting reason is reproductive dysfunction, infertility, and menstrual abnormality. However there is increasing evidence that PCOS is a risk factor for diabetes. A long-term prospective follow-up of women with PCOS showed that type 2 diabetes incidence was 1.95 per 100 person-years [8]. It was $\mathrm{a} \geq 10$-year follow-up of 255 women with PCOS. The risk of developing diabetes in the women was increased by the higher levels of body mass index and fasting glucose at baseline [8]. Similar risk of type 2 diabetes was observed even in young women with PCOS: data derived from the General Practice Research Database based in the UK (PCOS n:21,740) showed crude rates of diabetes of 5.7 per 1000 patient-years as compared to 1.7 in controls [9].

A position statement by the Androgen Excess Society recommended that [10] all women with PCOS be screened with a 2-h oral glucose tolerance test; those with normal response should be screened at least every 2 years or more often, in the presence of other risk factors. Those with 
impaired glucose tolerance must be screened every year for the development of diabetes. Both groups should be advised intensive lifestyle modifications to delay or prevent the onset of diabetes.

There are ethnic differences in the manifestations and risk factors for adverse outcomes from PCOS were reported. A report from the USA showed that the presence of diabetes in first-degree relatives increased the risk for impaired glucose tolerance or type 2 diabetes in women with PCOS [11]. In India, a comparison of lean versus obese women with PCOS from India showed that obese women with metabolic risk factors developed diabetes and endometrial hyperplasia at a younger age [12].

A community-based study on young women from Lucknow (age range 18-25) showed that PCOS had a calculated prevalence of $3.7 \%$; women were lean but had abdominal obesity [13], a marker of insulin resistance. Zargar et al. reported that women with type 2 diabetes commonly had ultrasonographic features of PCOS, nearly twice as common (61\%) compared to controls (36.7\%) [14]. In a hospital-based report from Maharastra, women with PCOS most often presented for reproductive and or cosmetic reasons in both lean and obese groups [15].

Intimal medial thickness is a risk factor of cardiovascular disease. We studied the intimal medial thickness in a group of 40 women with PCOS from southern India and compared with age- and weight-matched controls. This young group $(24 \pm 4.9$ years) was insulin resistant and had greater intimal medial thickening [16]. The difference could be accounted for differential fat distribution or by other rheological factors found in insulin resistance [16]. A recent study showed that insulin resistance was more often seen in classic presentation of PCOS and less so in the normoandrogenic phenotype [17].

Differences in phenotypic expression may be related to hyperandrogenism, hyperinsulinemia, and associated metabolic abnormalities, which may be responsible for differences in ethnic presentation of PCOS [18]. Underlying coexistent conditions such as thyroid disorders $[19,20]$ or use of medications [21] may also result in a clinical picture suggestive of PCOS. In addition, psychosocial stress has been shown to be significant in women with PCOS, a contributor to the development of diabetes and its management $[22,23]$. Biobehavioral mechanisms may operate in PCOS through insulin resistance, obesity, inflammation, and depression [24]. Consequently interventions must address psychological and behavioral aspects in PCOS.

Recent studies from India have shown that women with PCOS had insulin resistance as assessed by fasting glucose to insulin ratio [25]. The current issue of the journal addresses the issue of glucose intolerance and insulin resistance in a cohort of women with PCOS from southern India [26]. The prevalence of impaired glucose tolerance was found in one fourth of 45 women, diabetes in $2.2 \%$, and normal glucose tolerance in $69 \%$. They had higher levels of total cholesterol, triglycerides, and VLDL cholesterol and lower HDL, all precursors of future vascular disease from dysglycemia.

The question therefore arises is, PCOS being associated with the young, and presenting with reproductive disorders, does it go away with cessation of reproductive capacity? This is a valid point to be considered because of the associated metabolic abnormalities which increase with age. Is the reproductive capacity a concomitant of metabolic dysfunction? In other words, once reproduction ceases, do the metabolic disturbances still continue to manifest? This is important not only from the point of view of an individual subject but also could give insights into the underlying pathogenic processes. This question was recently addressed in a review of PubMed, bibliography from Evidence-Based PCOS Workshop, and the citations in them [27]. Women who have PCOS during reproductive years continue to have cardiovascular risk factors even after the menopause. Therefore menopause is not protective for cardiovascular risk in women who had PCOS.

Attention was also focused on the genetic component of PCOS. From the early approach of candidate gene studies, currently genome-wide association studies were performed [28]. There was evidence for association of PCOS and two genetic loci, $2 \mathrm{p} 16.3$ and $9 \mathrm{q} 33.3$, providing new insights into understanding its pathogenesis [28]. Further analysis showed eight new risk loci: 9q22.32, 11q22.1, 12q13.2, 12q14.3, $16 \mathrm{q} 12.1,19 \mathrm{p} 13.3$, and 20q13.2, along with a second independent signal at 2p16.3 [29]. A recent comprehensive interrogation of available genome-wide association results with in silico analysis suggests that the genetic basis of PCOS is considered the founder effect altered with human migration across generations. The prevalence supports "intralocus sexual conflict as alternative to the natural selection of phenotypic traits in females" [30].

Thus polycystic ovary syndrome, most well known for its cosmetic and reproductive abnormalities, nurses metabolic abnormalities which must be addressed. While subjects do not often present for insulin resistance, when the next woman comes with an extra hair on the chin or with abnormal menstrual cycles, consider PCOS. More than diagnosis and diagnostic criteria, preventive counseling against future metabolic complications is in order.

\section{References}

1. Casarini L, Brigante G. The polycystic ovary syndrome evolutionary paradox: a genome-wide association studies-based, in silico, evolutionary explanation. J Clin Endocrnol Metab. 2014;99:E2412-20.

2. Allahbadia GN, Merchant R. Polycystic ovary syndrome and impact on health. Middle East Fertility Soc J. 2011;16:19-37.

3. Ganie MA, Nisar S. Current perspectives of polycystic ovary syndrome (PCOS). JIMSA. 2008;21:161-5. 
4. Conway G, Dewailly D, Kandarakis ED, Morreale HFE, Franks S, Gambineri A, et al. The polycystic ovary syndrome: a position statement from the European Society of Endocrinology. Eur J Endocrinol. 2014;171:1-29.

5. Sridhar GR, Lakshmi G. Sleep and obesity. J Gen Med. 2009;21:54 6.

6. Buxton OM, Cain SW, O'Connor SP, Porter JH, Duffy JF, Wang W, et al. Adverse metabolic consequences in humans of prolonged sleep restriction combined with circadian disruption. Sci Transl Med. 2012;4:129ra-43.

7. Sridhar GR, Venkata P. Sleep and body weight in diabetes mellitus: a large retrospective analysis from south India. Diab Res Clin Pract. 2006;72:209-11.

8. Gambineri A, Patton L, Altieri P, Pagotto U, Pizzi C, Manzoli L, et al. Polycystic ovary syndrome is a risk factor for type 2 diabetes. Diabetes. 2012;61:2369-74.

9. Morgan CL, Jones SJ, Currie CJ, Rees DA. Evaluation of adverse outcome in young women with polycystic ovary syndrome versus matched, reference controls: a retrospective, observational study. J Clin Endocrinol Metab. 2012. doi:10.1210/jc.2012-1690.

10. Salley KES, Wickham EP, Cheang KI, Essah PA, Karjane NW, Nestler JE. Position statement: glucose intolerance in polycystic ovary syndrome - a position statement of the Androgen Excess Society. J Clin Endocrinol Metab. 2007;92:4546-56.

11. Ehrmann DA, Kasza K, Azziz R, Legro S, Ghazzi MN, for the PCOS/Troglitazone Study Group. Effects of race and family history of type 2 diabetes on metabolic status of women with polycystic ovary syndrome. J Clin Endocrinol Metab. 2005;90:66-71.

12. Majumdar A, Singh TA. Comparison of clinical features and health manifestations in lean vs obese Indian women with polycystic ovarian syndrome. J Hum Reprod Sci 2009;2:doi 10.4103/0974-1208. 51336

13. Gill H, Tiwari P, Dabadghao P. Prevalence of polycystic ovary syndrome in young women from North India: a community-based study. Indian J Endocrinol Metab. 2012;16 Suppl 2:S389-92.

14. Zargar AH, Gupta K, Wani AI, Masoodi S, Bashir MI, Laway BA, et al. Prevalence of ultrasonography proved polycystic ovaries in North Indian women with type 2 diabetes mellitus. Reprod Biol Endocrinol. 2005;3:35.

15. Ramanand SJ, Ghongane BB, Ramanand JB, Patwardhan MH, Ghanghas RR, Jain SS. Clinical characteristics of polycystic ovary syndrome in Indian women. Indian J Endocrinol Metab. 2013;17: 138-45.

16. Sundararaman PG, Manomani R, Sridhar GR, Sridhar V, Sundaravalli A, Umachander M. Risk of atherosclerosis in women with polycystic ovary syndrome: a study from south India. Metab Syndr el Dis. 2004;1:271-5.
17. Moghetti P, Tosi F, Bonin C, Sarra DD, Fiers T, Kaufman JM, et al. Divergence in insulin resistance in insulin resistance between the different phenotypes of the polycystic ovary syndrome. J Clin Endocrinol Metab. 2013;98:E628-37.

18. Jie Q. Pay more attention to ethnic differences in polycystic ovary syndrome phenotypic expression. Chinese Med J. 2013;126:2003-6.

19. Ganie MA, Marwaha RK, Aggarwal R, Singh S. High prevalence of polycystic ovary syndrome characteristics in girls with euthyroid chronic lymphocytic thyroiditis: a case-control study. Eur J Endocrinol. 2010;162:1117-22.

20. Ganie MA, Laway BA, Wani TA, Zargar MA, Nisar S, Ahamed F, et al. Association of subclinical hypothyroidism and phenotype, insulin resistance, and lipid parameters in young women with polycystic ovary syndrome. Fertile Steril. 2011;95:2039-43.

21. Sridhar GR, Nagamani G. Association of polycystic ovary syndrome with anticonvulsant use: a case report. Metabolic Syndr Rel Disorders. 2006;4:215-7.

22. Sundararaman PG, Shweta, Sridhar GR. Psychosocial aspects of women with polycystic ovary syndrome from south India. J Assoc Physicians India. 2008;56:945-8.

23. Sridhar GR, Madhu K. Stress in the cause and course of diabetes. Intl J Diab Dev Countries. 2001;21:112-20.

24. Farrell K, Antoni M. Insulin resistance, obesity, inflammation and depression in polycystic ovary syndrome: biobehavioral mechanisms and interventions. Fertil Steril. 2010;94:1565-74.

25. Kaviprasanna S, Saikumar P, Alaguveni T. Insulin resistance as an independent risk factor for the development of dyslipidemia in polycystic ovarian syndrome. Adv Biol Res. 2014;8:53-6.

26. Rajendra Prasad N, Bitla AR, Suchitra MM, Rajagopal G, Sachan A, Srinivasa Rao PVLN. A study on prevalence of abnormal glucose tolerance and correlation of insulin resistance with dyslipidemia in south-Indian women with polycystic ovary syndrome. Intl J Diab Dev Cntr 2014; 34: page numbers to be added

27. Welt CK, Carmina E. Lifecycle of polycystic ovary syndrome (PCOS): from in utero to menopause. J Clin Endocrinol Metab. 2013;98:4629-38.

28. Chen Z-J, Zhao H, He L, Shi Y, Qin Y, Shi Y, et al. Genome-wide association study identifies susceptibility loci for polycystic ovary syndrome on chromosome 2p16.3, 2p21 and 9q33.3. Nat Genet. 2011;43:55-60.

29. Shi Y, Zhao H, Shi Y, Cao Y, Yang D, Li Z, et al. Genome-wide association study identifies eight new risk loci for polycystic ovary syndrome. Nat Genet. 2012;44:1020-5.

30. Casarini L, Brigante G. The polycystic ovary syndrome evolutionary paradox: a genome-wide association studies-based, in silico, evolutionary explanation. J Clin Endocrinol Metab. 2014;99:E2412-20. 